\title{
Effects of NOX1 on fibroblastic changes of endothelial cells in radiation-induced pulmonary fibrosis
}

\author{
SEO-HYUN CHOI ${ }^{1}$, MISEON KIM ${ }^{1}$, HAE-JUNE LEE ${ }^{1}$, EUN-HO KIM ${ }^{2}$, CHUN-HO KIM ${ }^{3}$ and YOON-JIN LEE ${ }^{1}$ \\ ${ }^{1}$ Division of Radiation Effects; ${ }^{2}$ Division of Heavy Ion Clinical Research; ${ }^{3}$ Laboratory of Tissue Engineering, \\ Korea Institute of Radiological and Medical Sciences, Seoul 139-706, Republic of Korea
}

Received February 24, 2015; Accepted December 3, 2015

DOI: $10.3892 / \mathrm{mmr} .2016 .5090$

\begin{abstract}
Lung fibrosis is a major complication in radiation-induced lung damage following thoracic radiotherapy, while the underlying mechanism has remained to be elucidated. The present study performed immunofluorescence and immunoblot assays on irradiated human pulmonary artery endothelial cells (HPAECs) with or without pre-treatment with VAS2870, a novel NADPH oxidase (NOX) inhibitor, or small hairpin (sh)RNA against NOX1, -2 or -4 . VAS2870 reduced the cellular reactive oxygen species content induced by 5 Gy radiation in HPAECs and inhibited phenotypic changes in fibrotic cells, including increased alpha smooth muscle actin and vimentin, and decreased CD31 and vascular endothelial cadherin expression. These fibrotic changes were significantly inhibited by treatment with NOX1 shRNA, but not by NOX2 or NOX4 shRNA. Next, the role of NOX1 in pulmonary fibrosis development was assessed in the lung tissues of C57BL/6J mice following thoracic irradiation using trichrome staining. Administration of an NOX1-specific inhibitor suppressed radiation-induced collagen deposition and fibroblastic changes in the endothelial cells (ECs) of these mice. The results suggested that radiation-induced pulmonary fibrosis may be efficiently reduced by specific inhibition of NOX1, an effect mediated by reduction of fibrotic changes of ECs.
\end{abstract}

\section{Introduction}

Lung complications, such as pneumonitis and fibrosis, frequently occur in the thoracic primary and metastatic tumors following thoracic radiotherapy $(\mathrm{RT})(1,2)$ and sets a limit to its use and dosage. However, the underlying molecular mechanisms of radiation-induced pulmonary fibrosis (RIPF) have remained elusive. Inflammatory cytokines, transforming

Correspondence to: Dr Yoon-Jin Lee, Division of Radiation Effects, Korea Institute of Radiological and Medical Sciences, 215-4 Gongneung-Dong, Nowon-Gu, Seoul 139-706, Republic of Korea E-mail: yjlee8@kirams.re.kr

Key words: radiation, pulmonary fibrosis, NOX1, endothelial cells growth factor (TGF)- $\beta$ and chronic reactive oxygen species (ROS) are known to contribute to RIPF. ROS generated in large quantities by irradiation-induced cellular damage and involved in various signaling pathways and DNA fragmentation, which induces apoptosis in the initial phase of tissue damage (3-5). In addition, radiation-induced late normal tissue injury, including RIPF, is thought to be caused by chronic oxidative stress and inflammation. Therefore, anti-oxidant enzymes have been suggested to ameliorate radiation-induced chronic injury to normal tissue (6-8).

Nicotinamide adenine dinucleotide phosphate (NADPH) oxidases (NOXs) generate ROS (9) and catalyze the transfer of electrons from cytosolic NADPH to molecular $\mathrm{O}_{2}$ via the membrane-bound catalytic NOX or dual oxidase sub-units (9). NOXs are implicated in the pathophysiology of several diseases; specifically, NOXs in endothelial cells (ECs) are involved in various vascular diseases (9). ECs express four isoforms of NOX: Superoxide-generating enzyme NOX1, NOX2, hydrogen peroxide-generating enzyme NOX4 and NOX5. NOX4 has been implicated in EC apoptosis during the development of bleomycin-induced lung fibrosis. It was also recently reported that a NOX inhibitor reduced RIPF through inducing airway epithelial-cell senescence (10).

Radiation-induced vascular damage has an important role in normal tissue injury. Furthermore, EC dysfunction is thought to be associated with thromboresistance, the inflammatory response and vascular fibrosis (11-13). The present study focused on the effects of NOXs on the fibroblastic changes in ECs during RIPF. A specific NOX isoform that regulates radiation-induced fibroblastic changes in ECs was identified and the therapeutic potential of NOX inhibition in RIPF was demonstrated.

\section{Materials and methods}

Cell culture and treatment. Human pulmonary artery endothelial cells (HPAECs) were obtained from PromoCell (Heidelberg, Germany). All cells were used within nine passages. Endothelial Cell Growth Medium 2 (PromoCell) was used for HPAEC culture. VAS2870 was purchased from EMD Millipore (Billerica, MA, USA). Lentiviral vectors containing small hairpin RNAs (shRNAs) targeting NOX1, -2 or -4 , along with a control shRNA (cat. nos. sc-43938-V, sc-35503-V, sc-41586-V, and sc-108080), were purchased 
from Santa Cruz Biotechnology, Inc. (Dallas, TX, USA). Transfection was performed using $5 \times 10^{4}$ infectious units of the respective vector for $6 \times 10^{5}$ cells. Lentiviral particles were directly added into cells in OPTI-MEM (Invitrogen; Thermo Fisher Scientific, Inc., Waltham, MA, USA), and cells were incubated for $5 \mathrm{~h}$ with gentle shaking every $30 \mathrm{~min}$. Following the addition of growth medium, cells were incubated for 2 3 days before further treatments. Cells were exposed to gamma rays derived from a $\left[{ }^{137} \mathrm{Cs}\right]$ source using GammaCell 3000 (Atomic Energy of Canada, Mississauga, OT, Canada) at a dose rate of $3.81 \mathrm{~Gy} / \mathrm{min}$.

Measurement of ROS levels. Following the indicated treatments, cells were incubated for $30 \mathrm{~min}$ with $1 \mu \mathrm{m}$ 2',7'-dichlorodihydrofluorescein diacetate $\left(\mathrm{H}_{2} \mathrm{DCFDA}\right.$; Invitrogen; Thermo Fisher Scientific, Inc.) or $2.5 \mu \mathrm{m}$ MitoSOX ${ }^{\mathrm{TM}}$ (Invitrogen) at $37^{\circ} \mathrm{C}$, and washed twice with phosphate-buffered saline (PBS). The samples were then re-suspended in $1 \mathrm{ml}$ PBS and analyzed using a BD FACScan flow cytometer (BD Biosciences, Franklin Lakes, NJ, USA).

Immunofluorescence staining. Following the indicated treatments, cells were fixed with $100 \%$ ice-cold acetone (Sigma-Aldrich, St. Louis, MO, USA) for $5 \mathrm{~min}$, washed three times with PBS (pH 7.3) and incubated with antibodies against $\alpha$-SMA (1:1,000; mouse monoclonal anti-human; cat no. A5228; Sigma-Aldrich) and CD31 (1:100; goat polyclonal anti human; cat no. sc-1506; Santa Cruz), VE-cadherin (1:100; mouse monoclonal anti-human; cat no. sc-9989; Santa Cruz), and FSP (1:100; rabbit polyclonal anti-human, cat no. ab27957; Abcam, Cambridge, UK) in PBS containing Tween 20 (PBST; Sigma-Aldrich) and 2\% bovine serum albumin (Sigma-Aldrich) for $3 \mathrm{~h}$. Subsequently, samples were incubated with Alexa 488-conjugated donkey anti-mouse (or rabbit) and Alexa 546-conjugated donkey anti-goat (or mouse) antibodies (1:250; cat no. A21202, A21206, A11056, and A10036 ; Thermo Fisher Scientific, Inc., Waltham, MA, USA) for $1 \mathrm{~h}$ and washed with PBS. Cell nuclei were labeled with DAPI (5 $\mu \mathrm{M}$; cat no. D9542; Sigma-Aldrich), and stained cells were imaged using a Zeiss confocal microscope (Carl Zeiss AG, Oberkochen, Germany; magnification, x400).

Western blot analysis. For Western blot analysis, cells were lysed with RIPA (radio immunoprecipitation assay) buffer [50 mM Tris- $\mathrm{HCl}$ (pH 7.5), $150 \mathrm{mM} \mathrm{NaCl}, 1 \% \mathrm{NP}-40,0.1 \%$ sodium dodecyl sulfate (SDS), and $1 \%$ sodium deoxycholate] supplemented with $1 \mathrm{mM} \mathrm{Na}_{3} \mathrm{VO}_{4}, 1 \mathrm{mM}$ dithiothreitol, $1 \mathrm{mM}$ phenylmethylsulfonyl fluoride, and protease inhibitor cocktail (EMD Millipore). The supernatants were collected after centrifugation at $16,000 \mathrm{xg}$ for $15 \mathrm{~min}$ at $4^{\circ} \mathrm{C}$. Protein concentrations were measured using Bio-Rad Protein Assay (Bio-Rad Laboratories, Inc., Hercules, CA, USA). The samples were boiled for $5 \mathrm{~min}$, and an equal amount of protein was separated using SDS-polyacrylamide gel electrophoresis and transferred onto BioTrace NT Nitrocellulose Transfer Membranes (Pall Corporation, Pensacola, FL, USA). The membranes were incubated overnight at $4^{\circ} \mathrm{C}$ with primary antibodies against $\operatorname{CD} 31(1: 1,000$; cat $=0$. sc-1506, goat polyclonal anti-human), vascular endothelial (VE)-cadherin (1:1,000; cat no. sc-9989, mouse monoclonal anti-human),
TGF- $\beta$-receptor kinase I (ALK5) (1:1,000; cat no. sc-398, rabbit polyclonal anti-human), vimentin (1:1,000; cat no. sc-6260, mouse monoclonal anti-human), intercellular adhesion molecule 1 (ICAM1; 1:1,000; cat no. sc-7891, mouse anti-human) (all from Santa Cruz Biotechnology, Inc.), $\alpha$-SMA (1:5,000; cat no. A5228; mouse monoclonal anti-human) and $\beta$-actin (1:5,000; cat no. A1978, mouse monoclonal anti-human). All antibodies were sourced from Sigma-Aldrich. The membranes were then incubated with HRP-conjugated donkey anti-goat, goat anti-mouse, and goat anti-rabbit secondary antibodies (1:4000; cat nos. sc-2020, $\mathrm{sc}-2005$, and sc-2004) for $1 \mathrm{~h}$ at room temperature. HRP activity was measured using Western Lightning Plus-ECL (PerkinElmer, Inc., Waltham, MA, USA) and protein band intensity was visualized on AGFA CP-BU Medical X-Ray Film (Agfa HealthCare NV, Gevaert, Belgium) and quantified using Image J software 1.45 (National Institutes of Health, Bethesda, MD, USA)

Reverse-transcription quantitative polymerase chain reaction $(R T-q P C R)$. Total RNA was extracted from cells using TRIzol reagent (MRC, Cincinnati, OH, USA) and $1 \mu \mathrm{g}$ of total RNA was used to synthesize cDNA with an Omniscript RT kit (Qiagen, Hilden, Germany) according to the manufacturer's protocol. Then, $2 \mu \mathrm{l}$ of cDNA was amplified with Solgent Taq (Solgent, Daejeon, Korea) kits in a total volume of $25 \mu \mathrm{l}$ according to the manufacturer's protocol. The primers for NOX1, NOX2, and NOX4 were synthesized by Integrated DNA Technologies, Inc. (Coralville, IA, USA). PCR products were detected in agarose gels containing $1 \mu \mathrm{g} / \mathrm{ml}$ ethidium bromide. The primer sequences used were as follows. NOX1 forward, 5'-TGGAGTGGCTTGCACC-3' and reverse, 5'-TGC TGCATGACCAACCTTTT-3'; NOX2 forward, 5' -TTTACA CTGACATCCGCCCC-3' and reverse, 5'-TGGGCCGTCCAT ACAAAGTC-3'; NOX4 forward, 5'-CGGGCTTCCACTCAG TCTTT-3' and reverse, 5'-TGATCCGAGGTAAGCCA-3'. PCR conditions were as follows : $95^{\circ} \mathrm{C}$ for $2 \mathrm{~min}$, followed by 35 cycles, denaturation at $95^{\circ} \mathrm{C}$ for $30 \mathrm{sec}$, annealing at $58^{\circ} \mathrm{C}$ for $30 \mathrm{sec}$, and extension at $72^{\circ} \mathrm{C}$ for $45 \mathrm{sec}$. PCR products were detected in $2 \%$ agarose gels containing $1 \mu \mathrm{g} / \mathrm{ml}$ ethidium bromide, and scanned by Gel Doc ${ }^{\mathrm{TM}}$ XR+ Imager System (Bio-Rad Laboratories, Inc.). Band intensities of PCR products were quantified using Image J software (version 1.45; National Institutes of Health, Bethesda, MD, USA). The $2^{-\Delta \Delta C a}$ method was used to calculate the band density (14).

Mice and irradiation. All procedures of the present study were approved by the Institutional Animal Care and Use Committee of the Korea Institute of Radiological and Medical Sciences (Seoul, Korea). Mice (weight, 25-30 g) were purchased from Orient Bio (Seoul, South Korea) and maintained in $12 \mathrm{~h}$ light $12 \mathrm{~h}$ dark cycle with access to food and water ad libitum. They were maintained in an atmosphere of $18-24^{\circ} \mathrm{C}$, with $40-60 \%$ humidity. A total of 28 mice were used (control group, $n=4 ; 25$ Gy group, $n=5,25$ Gy + NOX inhibitor group, $n=5$; repeated twice). Radiation was delivered using an X-RAD 320 platform (Precision X-ray, North Branford, CT, USA) as described previously (15). Whole lungs of 10-week-old male C57BL/6 mice were irradiated. An NOX1 VAS2870 inhibitor (EMD Millipore) was dissolved in 
dimethyl sulfoxide (Sigma-Aldrich), further diluted in distilled water and administered intraperitoneally $(25 \mathrm{mg} / \mathrm{kg}) 1 \mathrm{~h}$ prior to thoracic irradiation with $25 \mathrm{~Gy}$; this was repeated twice at 1-day intervals.

Tissue histology and immunohistochemical staining. Mice were sacrificed by anesthesia with $\mathrm{CO}_{2}$. The lung tissue was harvested and fixed in $10 \%$ (v/v) neutral buffered formalin (Sigma-Aldrich) prior to preparation of paraffin (Sigma-Aldrich)-embedded samples, which were cut into 3-4 $\mu \mathrm{m}$ sections. To detect collagen, sections were de-paraffinized in xylene (Duksan Pure Chemicals Co., Ltd., Ansan, South Korea) and an ethanol series (95, 90, 70 and 50\%; Duksan Pure Chemicals Co., Ltd.), followed by washing with phosphate-buffered saline (PBS; Welgene, Inc., Gyeongsan-si, South Korea). De-paraffinized slides were boiled in $0.1 \mathrm{M}$ citrate buffer ( $\mathrm{pH}$ 6.0; Target retrival solution; Dako, Glostrup, Germany) for $20 \mathrm{~min}$ and incubated in 3\% hydrogen peroxide (Sigma-Aldrich) for $20 \mathrm{~min}$. Slides were then stained using a Masson's Trichrome Stain kit (Sigma-Aldrich) as described previously (16). Images were obtained using a Zeiss microscope (Carl Zeiss AG).

For immunofluorescence assays, de-paraffinized slides were boiled in $0.1 \mathrm{M}$ citrate buffer ( $\mathrm{pH}$ 6.0) for $20 \mathrm{~min}$ and incubated with $3 \%$ hydrogen peroxide for $20 \mathrm{~min}$. Slides were co-immunostained overnight at $4{ }^{\circ} \mathrm{C}$ with a goat polyclonal anti-human CD31 antibody (cat no. sc-1506; 1:100 dilution; Santa Cruz Biotechnology, Inc.) and rabbit polyclonal anti-human alpha smooth muscle actin $(\alpha-$ SMA) mAb (cat no. ab5694; 1:100 dilution; Abcam), followed by incubation for $1 \mathrm{~h}$ at room temperature with donkey anti-goat Alexa 488- or donkey anti-rabbit Alexa 546-conjugated secondary antibodies (1:250; cat no. A11055 and A10040; Thermo Fisher Scientific, Inc.). Cell nuclei were labeled with DAPI (5 $\mu \mathrm{M}$; cat no. D9542; Sigma-Aldrich). Images were captured using a Zeiss confocal microscope. Image $\mathbf{J}$ software (version 1.45) was used for quantitative evaluation of the staining.

Statistical analysis. Values are expressed as the mean \pm standard deviation of at least three independent experiments. Student's $t$-tests and analysis of variance were used to explore the statistical significance of differences between experimental groups. Statistical analyses were performed using GraphPad Prism software (version 5.0; GraphPad Software, Inc., La Jolla, CA, USA). $\mathrm{P}<0.05$ was considered to indicate a statistically significant difference.

\section{Results}

NOX inhibition reduces the generation of ROS in HPAECs. It has been suggested that NOXs have important roles in various pathological processes, including hypertension, cardiovascular disease and stroke $(9,17)$. The present study therefore assessed whether ROS production during radiation-induced lung damage was accountable for radiation-induced late normal tissue injuries including atherosclerosis and fibrosis in RIPF. Fluorescence-activated cell sorting using a DCF dye indicated that NOX inhibition reduced radiation-induced ROS in HPAECs (Fig. 1A). At $72 \mathrm{~h}$ after irradiation with $5 \mathrm{~Gy}$, the ROS content was elevated
$>2.0$ fold, which was, however, significantly attenuated in the group treated with the NOX inhibitor VAS28701 $(1 \mu \mathrm{m})$.

NOX inhibition reduces radiation-induced fibrotic changes in HPAECs. As shown in Fig. 1B, immunofluorescence analysis revealed that radiation increased the expression of $\alpha$-SMA, a fibroblastic cell marker, while decreasing the expression of EC marker CD31. Of note, these effects were inhibited by treatment with NOX inhibitor VAS2870. The endothelial-specific adhesion molecule CD31 was localized on the membranes of un-irradiated HPAECs and disappeared as the cells underwent fibroblastic changes, such as the endothelial-to-mesenchymal transition, in response to irradiation (Fig. 1B).

Consistent with this finding, western blot analysis showed increased expression of the fibrotic markers $\alpha$-SMA and vimentin, and decreased expression of the EC markers CD31 and VE-cadherin 3 days after 5-Gy radiation in HPAECs, which was significantly inhibited by VAS2870 treatment (Fig. 1C). These results indicated that NOX inhibition reduces ROS production and thus radiation-induced fibroblastic changes in HPAECs.

shRNA-mediated knockdown of NOX1, -2 and -4 decreases radiation-induced $R O S$ in HPAECs. To determine which NOX isoform regulates the radiation-induced ROS generation in ECs, their expression was determined by RT-qPCR. Irradiation increased the expression levels of NOX1, -2 and -4 on day 3 after 5-Gy radiation and to a lesser extent on days 5 and 7 (Fig. 2A). For further mechanistic study, HPAECs were transfected with NOX1, -2 or -4 shRNA. The decreased expression of the respective NOXs demonstrated the transfection efficiency of shRNA against NOX1, -2 or -4 (Fig. 2B). NOX1 shRNA specifically decreased radiation-induced NOX1 expression, while NOX2 and -4 shRNAs inhibited the expression of NOX2 as well as NOX4, indicating that NOX2 and -4 are cross-regulated. The effects of NOX knockdown on irradiation-induced ROS levels were then assessed. The level of intracellular ROS and mitochondrial superoxide (detected by H2DFHDA and mitSOX dye, respectively) in irradiated HPAECs were reduced by pre-treatment with NOX1, -2 or 4 shRNA to a similar extent (Fig. 2C and D). These results showed that knockdown of NOX1, -2 and -4 decreased radiation-induced ROS generation in HPAECs; furthermore, NOX1-targeted shRNA was indicated to be a more specific regulator than NOX2 or NOX4 shRNA.

NOX1 shRNA decreases radiation-induced fibrotic changes in HPAECs. Next, the present study aimed to identify the NOX isoform that is accountable for radiation-induced fibrotic changes in HPAECs. In HPAECs transfected with control shRNA, fibrotic changes were observed, including increased expression levels of $\alpha$-SMA and vimentin as well as decreased CD31 expression (Fig. 3A). NOX1 shRNA more significantly inhibited radiation-induced $\alpha$-SMA compared with NOX2 or -4 shRNA (Fig. 3A). In addition, immunofluorescence analysis showed that the irradiation-induced increase in fibroblast-specific protein 1 (FSP1), a fibroblastic cell marker, and the decrease in VE-cadherin were inhibited by NOX1 knockdown (Fig. 3B). 
A
(-) VAS2870

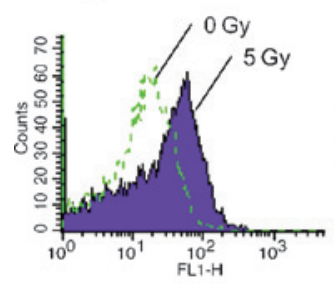

(+) VAS2870

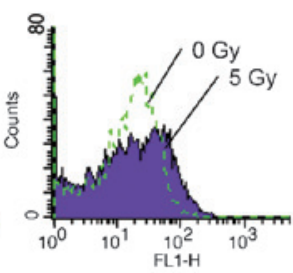

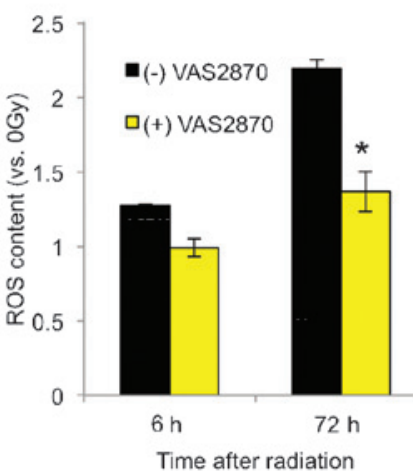

B

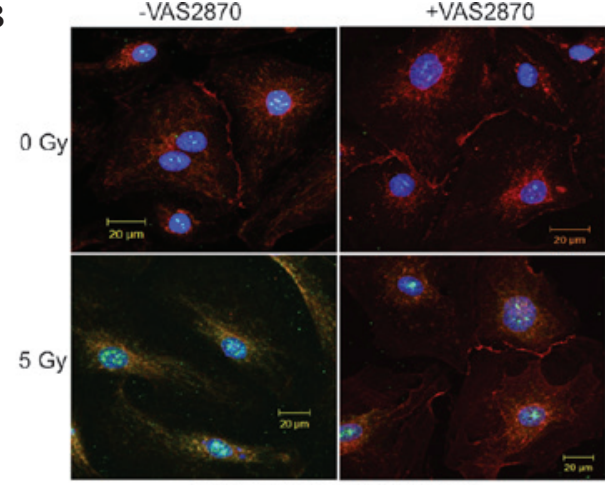

CD31 (Red) / aSMA(Green)
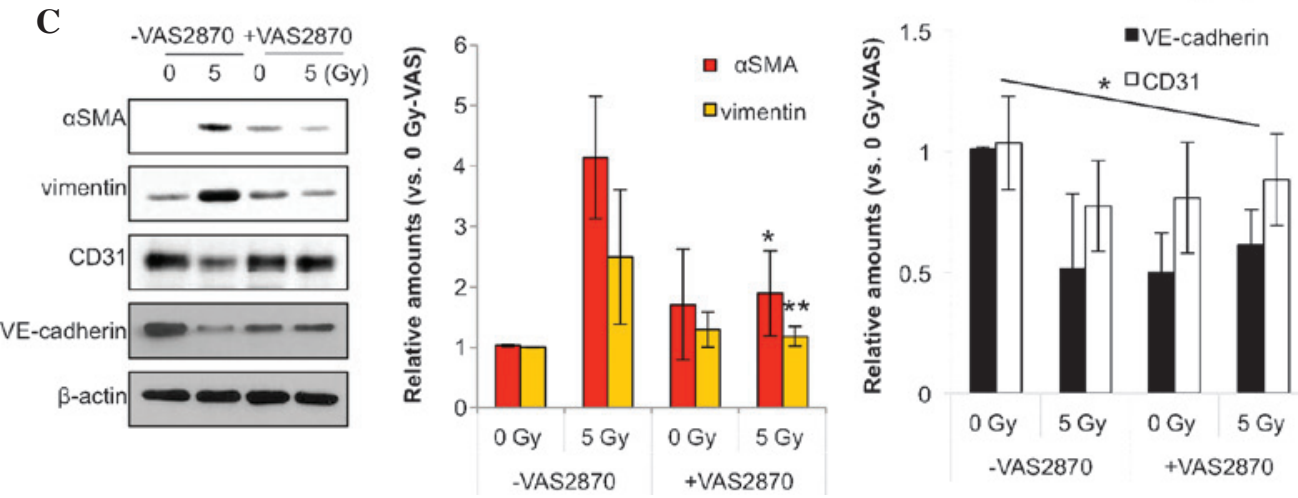

Figure 1. VAS2870 inhibits radiation-induced fibroblastic changes in ECs. (A) HPAECs were irradiated with 5 Gy and incubated for $72 \mathrm{~h}$. VAS2870 (1 $\mu \mathrm{m})$ was added to cells $1 \mathrm{~h}$ prior to irradiation. To measure ROS, cells were incubated for $30 \mathrm{~min}$ with $1 \mu \mathrm{m} \mathrm{2}, 7^{\prime}$-dichlorodihydrofluorescein diacetate and analyzed by flow cytometry ("P<0.05 vs. no VAS2870). (B) HPAECs were irradiated with 5 Gy and incubated for $72 \mathrm{~h}$. VAS2870 $(1 \mu \mathrm{m})$ was added to cells $1 \mathrm{~h}$ prior to irradiation and analysis by immunofluorescence with Alexa 488-conjugated anti- $\alpha$-SMA and Alexa 594-conjugated anti-CD31 antibodies (green and red, respectively). Nuclei were counterstained with 4',6-diamidino-2-phenylindole (blue) (scale bars, $20 \mu \mathrm{m}$ ). (C) Samples were subjected to western blot analysis of $\alpha$-SMA, vimentin, CD31 and VE-cadherin. $\beta$-actin served as the loading control. Protein expression was quantified by densitometric analysis. Values are expressed as the mean \pm standard deviation $(\mathrm{n}=3)$. ${ }^{*} \mathrm{P}<0.05$ and ${ }^{* *} \mathrm{P}<0.01$ vs. VAS2870-untreated. HPAEC, human pulmonary artery endothelial cell; SMA, smooth-muscle actin; VAS, nicotinamide adenine dinucleotide phosphate oxidase inhibitor VAS2870; ROS, reactive oxygen species; VE, vascular endothelial.

ALK5 expression was markedly increased at $4 \mathrm{~h}$ post-irradiation and peaked after 1 day, followed by a gradual decline until day 6 , while the $10-$ Gy dose had a greater effect than the 5-Gy dose (Fig. 4A). ICAM-1 levels were increased from $4 \mathrm{~h}$ on day 2 through day 4 until day 7 , corresponding to the transient and late responses. ALK5-associated signaling has been reported to mediate changes in the EC phenotype (18). The elevated ALK5 levels may also have been associated with the increased levels of the fibroblastic markers $\alpha$-SMA and vimentin. Furthermore, as shown in Fig. 4A, radiation (5 or $10 \mathrm{~Gy}$ ) induced an increase in the levels of ICAM-1, indicating EC activation and a phenotypic change. Increased ICAM-1 levels appeared as a dual regulation from $4 \mathrm{~h}$ to day 2 and from days 4-7. To assess the role of NOX in the radiation-induced increases in ALK5, HPAECs were pre-treated with VAS2870. The results showed that the increases in ALK5 levels caused by irradiation were markedly attenuated by VAS 2870 (Fig. 4B). In addition, HPAECs were pre-treated with NOX1, -2 or -4 shRNA prior to irradiation. As shown in Fig. 4C, pre-treatment with NOX1 shRNA significantly inhibited irradiation-induced increases in ALK5 expression (Fig. 4C). These results suggested that NOX1 is a molecule involved in the regulation of radiation-induced fibrotic changes in ECs via ALK5 signaling. ROS generated by NOX1 may contribute to fibrotic changes in irradiated ECs.
NOX1 inhibition reduces radiation-induced collagen deposition in the lung. An in vivo experiment was employed to demonstrate that an NOX1-specific inhibitor reduced radiation-induced collagen deposition during the development of RIPF (Fig. 5A). C57BL/6 mice received 25-Gy irradiation to the thoracic region with or without pre-treatment with NOX1-specific inhibitor. In inhibitor-pre-treated animals, the NOX1 inhibitor was further administered twice at 2-day intervals by intraperitoneal injection. Four weeks after irradiation, collagen deposition in the irradiated lung tissues was analyzed by trichrome staining. As shown in Fig. 5B, collagen deposition was increased in the irradiated lung tissues, while pre-treatment with NOX1 inhibitor significantly decreased collagen deposition.

To further examine fibroblastic changes in the ECs of the irradiated lung tissues, immunofluorescence assays were performed. Similar to the in vitro data, $\alpha$-SMA was upregulated and co-localized with CD31 in the ECs of irradiated lung tissues, indicating fibrotic changes (Fig. 5C). In addition, the NOX1 inhibitor abrogated the increases in $\alpha$-SMA expression in these ECs (Fig. 5C). It was therefore suggested that the observed fibrotic changes in the irradiated lung tissues may have contributed to increased collagen deposition. Furthermore, these results indicated that endothelial NOX1 inhibition can specifically diminish RIPF via attenuation of fibroblastic changes in irradiated ECs. 
A

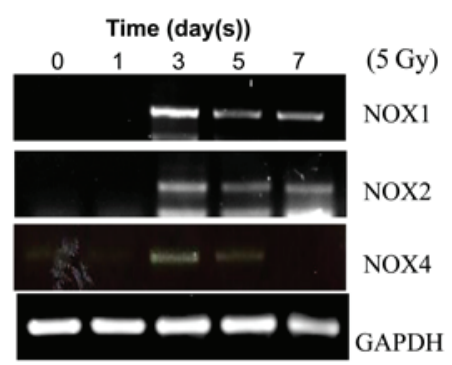

C

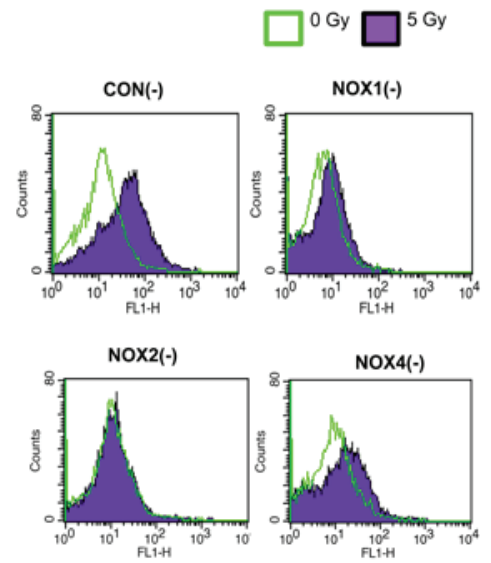

B

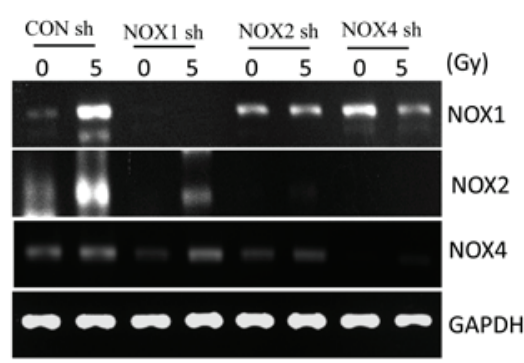

D

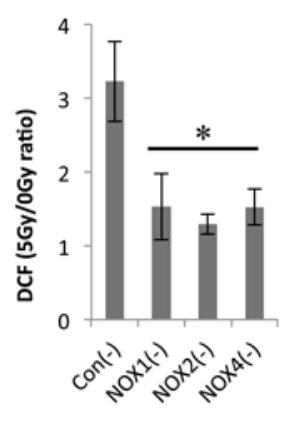

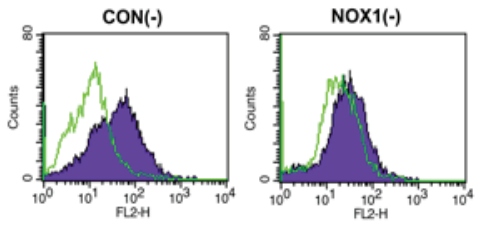
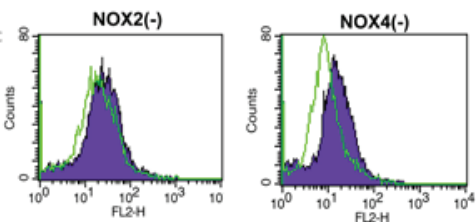

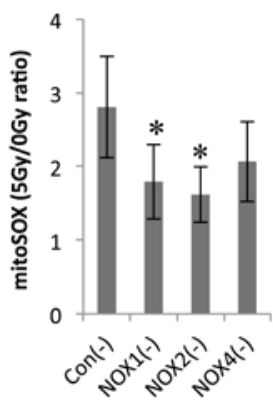

Figure 2. NOX1, 2 and 4 shRNAs decrease radiation-induced ROS in HPAECs. (A) Following irradiation, NOX1, 2 and 4 expression was evaluated by RT-qPCR. HPAECs were cultured for the indicated number of days after receiving 5 Gy irradiation. (B) Each lentiviral vector contained a NOX1-, 2- or 4-targeted shRNA, which was then transfected into cultured HPAECs. A lentiviral vector containing a scrambled sequence served as the control. To confirm lentiviral-mediated gene knockdown, NOX1, 2 and 4 expression was analyzed by RT-qPCR. (C) Assessment of ROS and (D) determination of mitochondrial superoxide. Infected cells were irradiated with $5 \mathrm{~Gy}$, followed by incubation with $1 \mu \mathrm{m} \mathrm{H}_{2}$ DCFDA or $2.5 \mu \mathrm{m}$ mitoSOX ${ }^{\mathrm{TM}}$, respectively, for 30 min and flow cytometric analysis. Values are expressed as the mean \pm standard deviation. ${ }^{*} \mathrm{P}<0.05(\mathrm{n}=6)$ in $\mathrm{C}$; $\mathrm{P}=0.05(\mathrm{n}=3)$ in $\mathrm{D}$ vs. control shRNA. HPAEC, human pulmonary artery endothelial cell; NOX, nicotinamide adenine dinucleotide phosphate oxidase; ROS, reactive oxygen species; GAPDH, glyceraldehyde 3-phosphate dehydrogenase; shRNA, small hairpin RNA; CON, control; RT-qPCR, reverse-transcription quantitative polymerase chain reaction; $\mathrm{H}_{2} \mathrm{DCFDA}$, 2',7'-dichlorodihydrofluorescein diacetate.

A

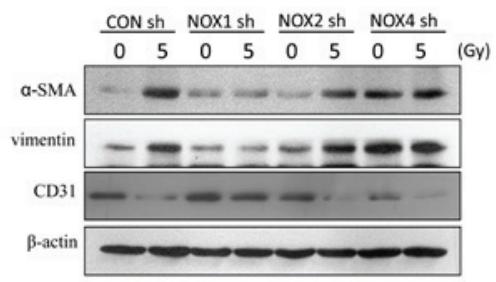

B

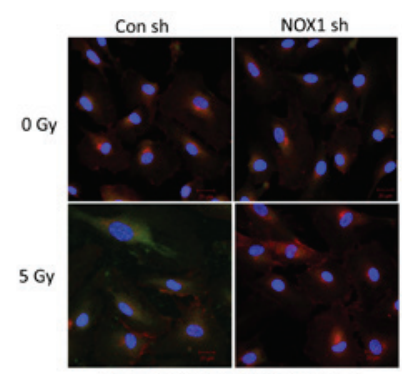

VE-cadherin (Red)/ FSP1(Green)
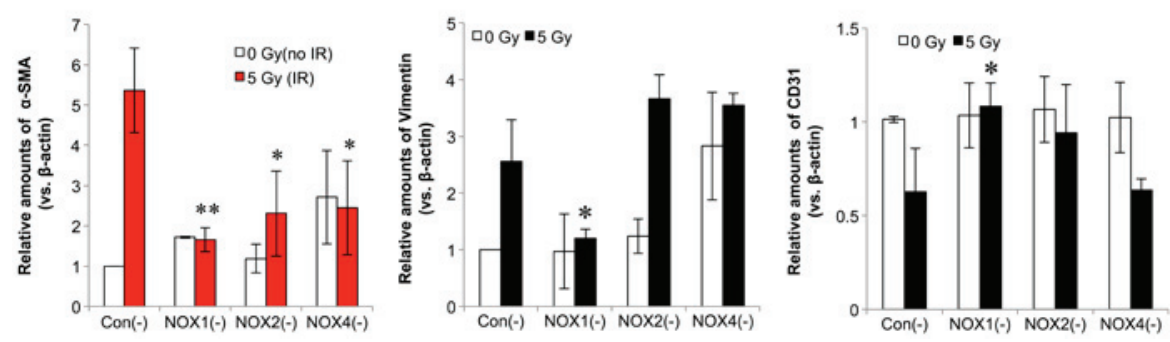

Figure 3. NOX1 shRNA decreases radiation-induced fibrotic changes in HPAECs. (A) Cells transfected with NOX1-, 2- or 4-targeted shRNA were irradiated and incubated for $72 \mathrm{~h}$, followed by western blot analysis of $\alpha$-SMA, vimentin and CD31. Protein levels were quantified by densitometric analysis of the blots. Values are expressed as the mean \pm standard deviation $(\mathrm{n}=3)$. ${ }^{* *} \mathrm{P}<0.005$; ${ }^{\mathrm{P}} \mathrm{P}<0.05, \alpha$-SMA vs. Con (-). (B) HPAECs transfected with NOX1 shRNA were irradiated with 5 Gy and incubated for $72 \mathrm{~h}$. Alexa 488-conjugated anti-FSP1 and Alexa 594-conjugated anti-VE-cadherin antibodies (green and red, respectively) were used to stain cells and nuclei were counterstained with 4',6-diamidino-2-phenylindole (blue). HPAEC, human pulmonary artery endothelial cell; SMA, smooth-muscle actin; VE, vascular endothelial; NOX, nicotinamide adenine dinucleotide phosphate oxidase; CON, control; shRNA, small hairpin RNA; IR, irradiation; FSP, fibroblast-specific protein. 
A

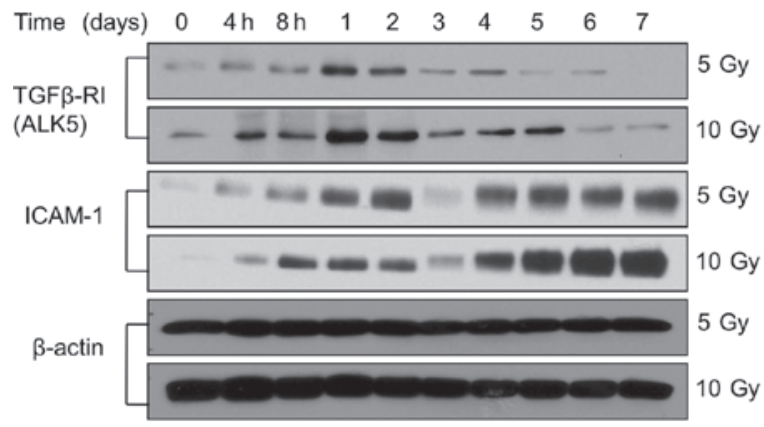

B

C

(Gy) $\frac{\mathrm{CON} \text { sh }}{0 \quad 5} \frac{\mathrm{NOX} 1 \mathrm{sh}}{0 \quad 5} \frac{\mathrm{NOX} 2 \mathrm{sh}}{0 \quad 5} \frac{\mathrm{NOX} 4 \mathrm{sh}}{0 \quad 5}$

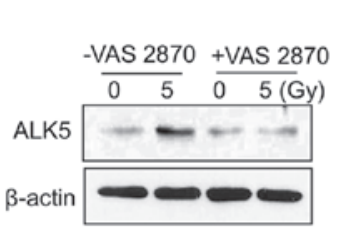

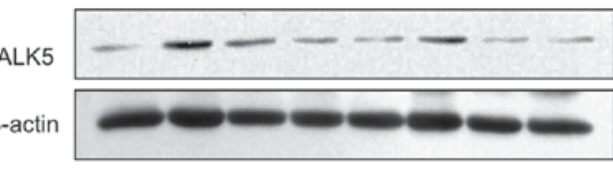

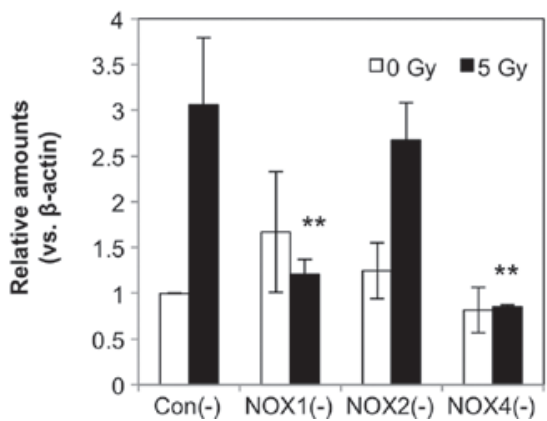

Figure 4. NOX1 shRNA reverses the radiation-induced increase in ALK5. (A) human pulmonary artery endothelial cells were irradiated with 5 or 10 Gy and incubated for 7 days. Cell lysates were used for western blot analysis of ALK5, ICAM-1 and $\beta$-actin. Cells treated with (B) VAS2870 or (C) transfected with NOX1-, 2- or 4-targeted shRNA were subjected to western blot analysis of ALK5 and $\beta$-actin. Protein levels were quantified by densitometric analysis of the blots. Values are expressed as the mean \pm standard deviation $(\mathrm{n}=3)$. ${ }^{* * *} \mathrm{P}<0.01$ ALK5 vs. Con (-). NOX, nicotinamide adenine dinucleotide phosphate oxidase; CON, control; shRNA, small hairpin RNA; TGF, transforming growth factor; ICAM, intercellular adhesion molecule; ALK5, TGF- $\beta$ type I receptor kinase.

A

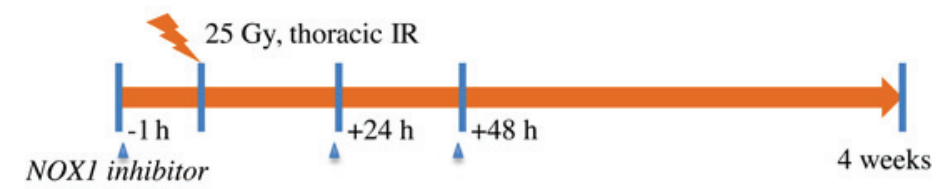

B

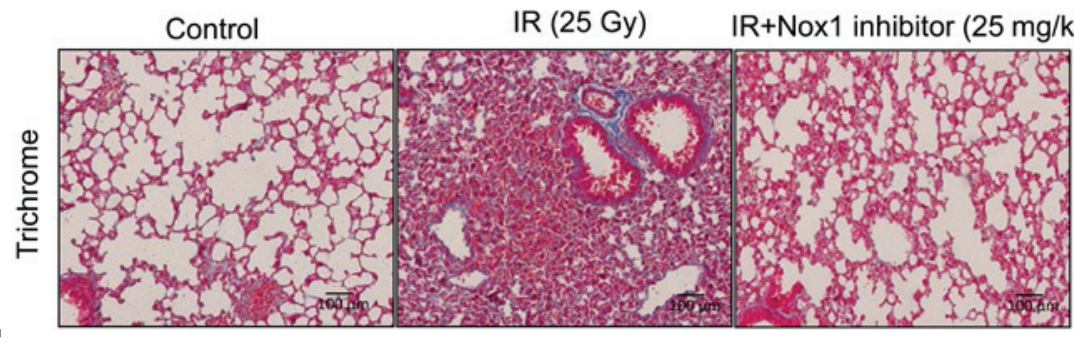

C
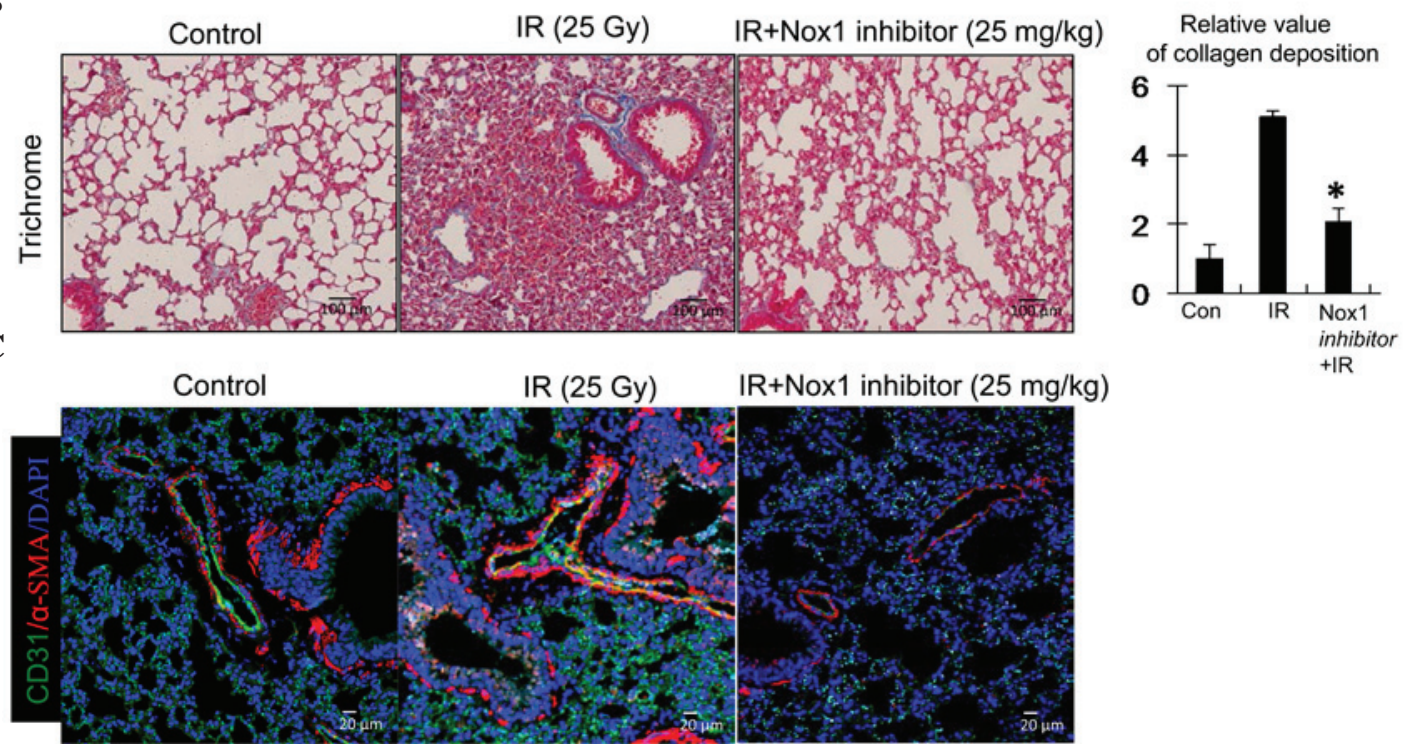

Figure 5. Collagen deposition and fibrotic changes in endothelial-cells of irradiated lung tissue. (A) Schematic illustrating the experimental design. C57BL/6 mice were subjected to thoracic irradiation with $25 \mathrm{~Gy}$. Lung samples ( $\mathrm{n}=4-5$ per condition) were obtained from mice prior to and 4 weeks after irradiation (B) Representative trichrome-stained images of collagen deposition. Collagen is stained blue, nuclei are purple and cytoplasm is red/pink (scale bar, $50 \mu \mathrm{m}$ ). The graph shows the relative area of collagen deposition in irradiated lung tissue, determined from determined from randomly selected microscopic field with Image J software (five field per mouse at X200 magnification). The relative levels of collagen deposition per 200x field are expressed as the mean \pm standard deviation ( $n=3)$; "P<0.05 vs. Nox1 inhibitor-untreated. (C) Fibroblastic changes in ECs in the irradiated samples were analyzed by immunofluorescence using Alexa 594-conjugated anti- $\alpha$-SMA and Alexa 488-conjugated anti-CD31 antibodies (red and green, respectively) with nuclei counterstained with DAPI. NOX, nicotinamide adenine dinucleotide phosphate oxidase; IR, irradiation; CON, control; DAPI, 4',6-diamidino-2-phenylindole.

\section{Discussion}

RIPF generally develops $\sim 6-24$ months following tissue damage and stabilizes after two years (1). This chronic complication is thought to be caused by chronic ROS accumulation $(6,19)$. Specifically, NOXs generate superoxide, a toxic type of ROS. Since NOXs are constitutively present in most cell types, NOX inhibitors are associated with toxic effects $(9,17)$. A 
recent study showed that NOX blocked the radiation-mediated upregulation of intracellular ROS in microvascular ECs of the rat brain, suggesting that NOX may be an important regulator of radiation-induced brain injury in patients with brain metastasis (20). Although NOX is an efficient target for regulating ROS in various diseases, including radiation-induced tissue damage, its clinical use is limited by the unpredictable side effects of non-selective NOX inhibition (21). Thus, the present study aimed to identify the specific NOX isoform that regulates RIPF. The role of NOX in RIPF has remained to be fully elucidated. Recently, Jarman et al (22) reported that an NOX4 inhibitor reduced idiopathic pulmonary fibrosis through a TGF- $\beta$-associated signaling mechanism.

Radiation-induced late normal tissue injuries including atherosclerosis and fibrosis are, in part, due to vascular compromise. A recent study by our group reported fibroblastic changes in vascular ECs in radiation-induced atherosclerosis (23). In addition, vascular damage and subsequent inflammation can occur during RIPF development (13). During RIPF development, radiation can disrupt the integrity of the pulmonary epithelium and endothelium, leading to edema and leukocyte recruitment, and resulting in alterations of the microenvironment (24).

Several studies have reported radiation-induced fibroblastic phenotypic changes in alveolar epithelial cells $(10,17)$. Furthermore, mesenchymal transition or senescence of alveolar epithelial cells are linked with the development of RIPF (10,25). However, the underlying molecular mechanisms of pathological changes in vascular ECs resulting in chronic fibrosis in radiation-induced lung injury have remained elusive. Adamson and Bowden (26) suggested that acute endothelial injury may be rapidly repaired with little stimulation of the fibroblasts, while more severe or prolonged injury with delayed regeneration disrupting the endothelial-mesenchymal balance. The endothelial-to-mesenchymal transition is thought to be mainly associated with diseases including cardiac fibrosis, kidney fibrosis, bleomycin-induced fibrosis and cancer $(19,22)$. However, the mechanism underlying the fibroblastic changes that occur in ECs during RIPF have remained elusive.

The present study identified fibroblastic changes in vascular HPAECs subjected to irradiation, which promoted collagen deposition. Specifically, NOX1 shRNA efficiently inhibited fibrotic changes in ECs compared with NOX2 and -4 shRNA in irradiated HPAECs. NOX1 has been reported to be expressed in vascular smooth muscle cells and ECs (9). Furthermore, NOX1 is more closely associated with EC dysfunction in ROS-induced acute injury than other NOX isoforms $(9,17,27)$. Several studies have reported that basal blood pressure, systemic hypertension and the early stage of atherosclerosis depend on NOX1 $(9,17,27)$. In the present study, as expected, a small NOX1-specific inhibitor significantly inhibited RIPF in vivo. Therefore, it is hypothesized that NOX1 is a specific target in the regulation of radiation-induced EC damage and subsequent development of RIPF. In addition, it is indicated that fibroblastic changes in irradiated ECs may be accountable for RIPF in the initial stage of radiation-induced lung damage.

\section{Acknowledgements}

The present study was supported by the Nuclear Research and Development Program (grant nos. NRF-2012M2A2A7012483,
NRF-2011-0031697 and NRF-2013M2A2A7043580) and a KIRAMS Research Project (grant no. 50520-2013) funded by the Nuclear Research and Development Program.

\section{References}

1. Mehta V: Radiation pneumonitis and pulmonary fibrosis in non-small-cell lung cancer: Pulmonary function, prediction and prevention. Int J Radiat Oncol Biol Phys 63: 5-24, 2005.

2. Fleckenstein K, Gauter-Fleckenstein B, Jackson IL, Rabbani Z, Anscher M and Vujaskovic Z: Using biological markers to predict risk of radiation injury. Semin Radiat Oncol 17: 89-98, 2007.

3. Ueno M, Maeno T, Nomura M,Aoyagi-Ikeda K, Matsui H,Hara K, Tanaka T, Iso T, Suga T and Kurabayashi M: Hypoxia-inducible factor- $1 \alpha$ mediates TGF- $\beta$-induced PAI-1 production in alveolar macrophages in pulmonary fibrosis. Am J Physiol Lung Cell Mol Physiol 300: L740-L752, 2011.

4. Rodemann HP and Bamberg M: Cellular basis of radiation-induced fibrosis. Radiother Oncol 35: 83-90, 1995.

5. Molteni A, Moulder JE, Cohen EF, Ward WF, Fish BL, Taylor JM, Wolfe LF, Brizio-Molteni L and Veno P: Control of radiation-induced pneumopathy and lung fibrosis by angiotensin-converting enzyme inhibitors and an angiotensin II type 1 receptor blocker. Int J Radiat Biol 76: 523-532, 2000.

6. Guerrero T, Martinez J, McCurdy MR, Wolski M and McAleer MF: Elevation in exhaled nitric oxide predicts for radiation pneumonitis. Int J Radiat Oncol Biol Phys 82: 981-988, 2012.

7. Zhang Y, Zhang X, Rabbani ZN, Jackson IL and Vujaskovic Z: Oxidative stress mediates radiation lung injury by inducing apoptosis. Int J Radiat Oncol Biol Phys 83: 740-748, 2012.

8. Fleckenstein K, Zgonjanin L, Chen L, Rabbani Z, Jackson IL, Thrasher B, Kirkpatrick J, Foster WM and Vujaskovic Z: Temporal onset of hypoxia and oxidative stress after pulmonary irradiation. Int J Radiat Oncol Biol Phys 68: 196-204, 2007.

9. Drummond GR and Sobey CG: Endothelial NADPH oxidases: Which NOX to target in vascular disease? Trends Endocrinol Metab 25: 452-463, 2014.

10. Citrin DE, Shankavaram U, Horton JA, Shield W III, Zhao S, Asano H, White A, Sowers A, Thetford A and Chung EJ: Role of type II pneumocyte senescence in radiation-induced lung fibrosis. J Natl Cancer Inst 105: 1474-1484, 2013.

11. Brush J, Lipnick SL, Phillips T, Sitko J, McDonald JT and McBride WH: Molecular mechanisms of late normal tissue injury. Semin Radiat Oncol 17: 121-130, 2007.

12. Yarnold J, Brotons MC: Pathogenetic mechanisms in radiation fibrosis. Radiother Oncol 97: 149-161, 2010.

13. Weintraub NL, Jones WK and Manka D: Understanding radiation-induced vascular disease. J Am Coll Cardiol 55: $1237-1239,2010$.

14. Schmittgen TD and Livak KJ: Analyzing real-time PCR data by the comparative $\mathrm{C}(\mathrm{T})$ method. Nat Protoc 3: $1101-1108$, 2008.

15. Hong ZY, Lee HJ, Choi WH, Lee YJ, Eun SH, Lee JI, Park K, Lee JM and Cho J: A preclinical rodent model of acute radiation-induced lung injury after ablative focal irradiation reflecting clinical stereotactic body radiotherapy. Radiat Res 182: 83-91, 2014.

16. Lee YJ, Koch M, Karl D, Torres-Collado AX, Fernando NT, Rothrock C, Kuruppu D, Ryeom S, Iruela-Arispe ML and Yoon SS: Variable inhibition of thrombospondin 1 against liver and lung metastases through differential activation of metalloproteinase ADAMTS1. Cancer Res 70: 948-956, 2010.

17. Sanders KA and Hoidal JR: The NOX on pulmonary hypertension. Circ Res 101: 224-226, 2007.

18. Ten Dijke P, Egorova AD, Goumans MJ, Poelmann RE and Hierck BP: TGF- $\beta$ signaling in endothelial-to-mesenchymal transition: The role of shear stress and primary cilia. Sci Signal 5: pt2, 2012

19. Mancini ML and Sonis ST: Mechanisms of cellular fibrosis associated with cancer regimen-related toxicities. Front Pharmacol 5: 51, 2014

20. Collins-Underwood JR, Zhao W, Sharpe JG and Robbins ME: NADPH oxidase mediates radiation-induced oxidative stress in rat brain microvascular endothelial cells. Free Radic Biol Med 45: 929-938, 2008 
21. Aldieri E, Riganti C, Polimeni M, Gazzano E, Lussiana C, Campia I and Ghigo D: Classical inhibitors of NOX NAD (P) H oxidases are not specific. Cur Drug Metab 9: 686-696, 2008.

22. Jarman ER, Khambata VS, Cope C, Jones P, Roger J, Ye LY, Duggan N, Head D, Pearce A, Press NJ, et al: An inhibitor of NADPH oxidase-4 attenuates established pulmonary fibrosis in a rodent disease model. Am J Respir Cell Mol Biol 50: $158-169,2014$

23. Kim M, Choi SH, Jin YB, Lee HJ, Ji YH, Kim J, Lee YS and Lee YJ: The effect of oxidized low-density lipoprotein (ox-LDL) on radiation-induced endothelial-to-mesenchymal transition. Int J Radiat Biol 89: 356-363, 2013.
24. Rubin P, Johnston CJ, Williams JP, McDonald S and Finkelstein JN: A perpetual cascade of cytokines postirradiation leads to pulmonary fibrosis. Int J Radiat Oncol Biol Phys 33: 99-109, 1995.

25. Almeida C, Nagarajan D, Tian J, Leal SW, Wheeler K, Munley M, Blackstock W and Zhao W: The role of alveolar epithelium in radiation-induced lung injury. PloS One 8: e53628, 2013.

26. Adamson IY and Bowden DH: Endothelial injury and repair in radiation-induced pulmonary fibrosis. Am J Pathol 112: 224-230, 1983.

27. Ray R and Shah AM: NADPH oxidase and endothelial cell function. Clin Sci (Lond) 109: 217-226, 2005. 